\title{
Efectos sobre la reincidencia de un programa de competencia psicosocial en jóvenes delincuentes
}

\section{Effects of a psychosocial competence program about recidivism in young offenders}

Recibido el 21 julio 2015/Publicado el 10 diciembre 2015

\author{
Antoni Forcadell ${ }^{1}$ y Jorge Pérez \\ Universidad Pompeu Fabra
}

\section{RESUMEN}

El objetivo del presente estudio consistió en determinar la posible efectividad de un programa básico en competencia psicosocial que era el más ampliamente aplicado en un centro de Cataluña. El estudio compara las tasas de reincidencia entre las personas que siguieron y que no siguieron el programa mientras estuvieron internados. Se analizó la tasa de reincidencia grave de 60 personas que habían estado internos en el centro, 26 que habían participado en el programa y 34 que no. Los resultados mostraron una tasa inferior de reincidencia en aquellos internos que habían seguido el tratamiento (19\%) respecto a los que no habían seguido el tratamiento (35\%).

Palabras clave: delincuentes juveniles, reincidencia, rehabilitación de delincuentes, programa de competencias psicosociales.

\footnotetext{
${ }^{1}$ Autor de correspondencia: Antoni Forcadell. Facultat de Dret. Universitat Pompeu Fabra. Campus de la Ciutadella, Edifici Roger de Llúria, Ramon Trias Fargas, 25-27, 08005 Barcelona. Spain. tforcadell@gencat.cat
} 


\begin{abstract}
The aim of this study is to analyse the possible effectiveness of a basic program in psychosocial competence that was the most widely applied in a detention centre in Catalonia. The study compared recidivism rates among people who followed and not followed the program while being in detention. The rate of serious recidivism of 60 people who had been inmates in the centre was analysed, 26 had participated in the program and 34 did not. The results showed a lower rate of recidivism for those inmates who had been on treatment (19\%) than those who had not followed the treatment (35\%).
\end{abstract}

Keywords: juvenile offenders, recidivism, offender rehabilitation, psychosocial competences program.

\title{
1. Introducción
}

La efectividad del tratamiento de delincuentes juveniles y adultos ha sido un tema de mucho interés tanto para los centros de rehabilitación como para la investigación científica. En los años 70 del pasado siglo, Martinson (1974) en un famoso y radical artículo afirmó que nada funcionaba (Nothing Works). La investigación posterior permitió evolucionar hacia un punto de vista más optimista que proponía que algunas cosas funcionan en algunos lugares con algunas personas (Antonowick \& Ross, 1994; Redondo, Garrido \& Sánchez-Meca, 1997). En los años posteriores y hasta la actualidad se ha generado bastante investigación que ha permitido tener una perspectiva bastante concreta sobre este tema.

Así Killias, Redondo \& Sarnecki (2012) concluyen, en relación a los delincuentes juveniles, que las revisiones europeas de tratamientos de delincuentes juveniles, las cuales según ellos son similares a las revisiones americanas, han mostrado que los tratamientos tienen un modesto pero significativo efecto en reducir la delincuencia. Estos autores concluyen de acuerdo a los datos de diversos metaanálisis que las intervenciones consiguen una reducción de la reincidencia media de unos 10 puntos porcentuales, pero que los mejores programas de tratamiento pueden conseguir reducciones de 15 a 25 puntos. Unos resultados similares obtiene Redondo (2008) en una revisión de 22 metaanálisis realizados entre el 1985 y 2006 sobre tratamientos de la delincuencia juvenil. 
Los programas de tratamiento que han probado ser más efectivos combinan varias técnicas, principalmente del modelo cognitvo-conductual (Andrews, Bonta \& Hoge, 1990; Redondo et al., 1997). Gran parte de estas técnicas están basadas en el programa pionero de Razonamiento y Rehabilitación de Ross \& Fabiano (1985). Con estas técnicas se busca fomentar las competencias siguientes: el hábito de reflexionar antes de actuar, la empatía, la perspectiva social, el pensamiento creativo y flexible, el locus de control interno, un razonamiento moral desarrollado, y las habilidades para resolver problemas sociales y interactuar con los demás, entre otras (Ross, Fabiano \& Garrido, 1990). Los mismos autores proponen para ello, entre otras cosas, el desarrollo del pensamiento creativo (De Bono, 1985), el entrenamiento en habilidades sociales (Goldstein, Sprafkin \& Gershaw, 1976), el desarrollo de valores (Galbraith \& Jones, 1976) y la técnica de resolución de problemas (Spivack, Platt \& Shure, 1976).

En los últimos años diversos metaanálisis han evaluado la eficacia de los programas de entrenamiento en competencia psicosocial basados en el modelo cognitivo-conductual. Todos ellos coinciden en mostrar la eficacia de estas técnicas en la reducción de las tasas de reincidencia tanto con jóvenes como con adultos. Así, Landerberger \& Lipsey (2005) en uno de los más completos metaanálisis sobre los efectos de la terapia cognitivo conductual sobre la reincidencia encontraron que estos programas conseguían una reducción media en las tasa de reincidencia del $22 \%$. Tong \& Farrington (2006) en un metaanálisis sobre la efectividad del programa sobre razonamiento y rehabilitación observaron una reducción media del 14\% en las tasas de reincidencia como consecuencia de haber participado en este programa.

En un metaanálisis más específico sobre la efectividad de las intervenciones rehabilitadores con delincuentes juveniles graves realizadas en centros de seguridad, Garrido, Morales \& Sánchez-Meca (2006) encontraron unos resultados similares. Observaron que la reducción media de la reincidencia era del $7 \%$ y que los programas cognitivo conductuales y cognitivos aparecían como los más efectivos.

Un aspecto importante para valorar la efectividad de los programas de tratamiento es, como contempla el Modelo R-N-R (Andrews, Zinger, Hoge, Bonta, Gendreau \& Cullen, 1990), la integridad en la aplicación de los programas. Algunos autores han contemplado esta variable eliminando del grupo experimental a aquellos sujetos que no habían finalizado el programa o bien no lo habían empezado. Siguiendo 
esta práctica, Cann, Falshaw \& Friendship (2005) con jóvenes delincuentes, Van Voorhis, Spruance, Johnson Listwan \& Seabrook (2004) y Hollin, McGuire, Hounsome, Hatcher, Bilby \& Palmer (2008) con delincuentes en la comunidad, han mostrado que las personas que completaban el programa cognitivo conductual tenían tasas de reincidencia inferiores a las que no lo habían completado o no lo habían llegado a empezar. Estos resultados serían una muestra más a favor de la eficacia de este tipo de técnicas y pondrían de relieve la importancia de poner en práctica esta estrategia metodológica.

En España las técnicas cognitivo conductuales se habían usado muy poco en los procesos de rehabilitación de delincuentes juveniles hasta los principios de los años 2000 a pesar de las evidencias a favorables a su utilización durante una década anterior. De hecho, en el metaanálisis referenciado sobre tratamientos de delincuentes juveniles graves, Garrido et al. (2006) informaban que casi todos los estudios habían sido realizados en los Estados Unidos.

Solamente tenemos conocimiento de tres investigaciones que hayan intentado evaluar la efectividad de este tipo de programas aplicados a delincuentes juveniles en centros españoles.

Garrido (1989) informaba de diversas pruebas piloto sobre la aplicación de un programa de entrenamiento en habilidades cognitivas y sociales aplicado en un centro de menores delincuentes. Este programa incluía las técnicas siguientes: Aprendizaje Estructurado de Habilidades Sociales (Goldstein et al., 1976), Técnica de Resolución de Problemas (Spivack et al., 1976), CORT (De Bono, 1985) y Razonamiento Moral (Galbraith \& Jones, 1976). El autor daba cuenta de la buena recepción de dichos programas en los profesionales y en los internos pero no presentaba datos de eficiencia ya que se estaban empezando a implementar.

Garrido, Redondo \& Pérez (1989) evaluaron un programa muy similar en la prisión de jóvenes "La Trinidad" en Barcelona. El programa contenía las mismas técnicas mencionadas en el parágrafo anterior y la técnica de inoculación de estrés (Meichenbaum, 1987). Se compararon los resultados obtenidos por un grupo experimental y por un grupo control en diversas pruebas psicológicas y en una medida conductual de comportamiento prosocial dentro de la prisión. En el análisis pre post dentro del grupo experimental se observó una mejora en la medida conductual de 
comportamiento prosocial $\mathrm{y}$ en las medidas psicológicas perspectiva social $\mathrm{y}$ pensamiento medios-fines. En la comparación entre grupo control y experimental postest se observó una mejora en el grupo experimental en pensamiento moral. No obstante, estos resultados se deben valorar con cautela, ya que el tiempo de funcionamiento del programa fue muy corto.

Redondo, Álvarez, Antequera, Cano \& Martínez (2010) se propusieron comprobar la eficacia de un programa cognitivo conductual de control de la violencia aplicado a jóvenes que cumplían medidas de Justicia Juvenil en medio abierto. También en este caso se compararon los resultados pre - post y del grupo control (17 sujetos) y experimental (11 sujetos) en seis medias psicológicas. Además se valoró la satisfacción de los usuarios con el programa, la cual resulto ser alta (de 20,88 sobre un máximo de 24). En relación a las variables psicológicas se observó en el grupo experimental respecto al control una mejora en autoestima y habilidades sociales y una reducción en agresividad.

Cataluña tiene un centro de justicia juvenil destinado al cumplimento de medidas de internamiento cerrado de larga duración. En éste cumplen la medida aquellos jóvenes condenados por delitos muy graves o multirreincidentes en delitos contra la propiedad. En dicho centro se empezaron a aplicar diversos programes cognitivo conductuales desde el año 1996. En el año 1999 se estaban aplicando los programas siguientes: programa básico de competencia psicosocial, educación en valores, desarrollo de la inteligencia, conductas adictivas y tratamiento del comportamiento violento.

A pesar de la satisfacción de los profesionales y algunas evaluaciones internas que mostraban efectos positivos sobre la conducta de los internos, no existían evidencias sobre los efectos de los programas sobre la reincidencia.

Nosotros en este trabajo nos hemos propuesto conocer la relación entre algunos de estos programas y la reincidencia. En concreto, dado que no conocemos que se haya realizado en España un estudio previo sobre el tema, hemos considerado la utilidad de estudiar el posible efecto de los programas de entrenamiento en competencia psicosocial sobre la reincidencia.

Así, el objetivo del presente estudio consiste en determinar la posible efectividad del programa básico en competencia psicosocial que era el más ampliamente aplicado en el centro. El interés del trabajo consiste en observar si las tasas de reincidencia de los 
internos que habían realizado el programa de competencia psicosocial serían diferentes de las de los que no habían participado en este programa.

\section{Método}

\subsection{Emplazamiento}

El estudio que se presenta se realizó en el "Centre Educatiu l'Alzina" de Palau de Plegamans i Solita, una población cercana a Barcelona. Se trata del centro cerrado de la Generalitat de Cataluña destinado al cumplimiento por parte de menores varones de penas largas dictadas por los jueces de menores. Por lo tanto, en él están internados delincuentes juveniles que han cometido delitos graves (entre otros, homicidios, agresiones sexuales) o delincuentes multirreincidentes contra la propiedad.

El funcionamiento del centro estaba organizado según un sistema de fases progresivas, separadas físicamente unas de otras.

Las fases iban desde una de alta seguridad, con un régimen muy restrictivo donde se ubicaba a los internos con comportamiento más desadaptado, a la última en la que se disfrutaba de un régimen de semilibertad destinada a aquellos internos con un mayor nivel de adaptación. El comportamiento adaptativo comportaba progresar a través del sistema de fases mientras que el comportamiento antisocial significaba regresar a fases anteriores.

A parte de este sistema de evaluación y motivación, existía un protocolo unificado de programas de tratamiento basado en el modelo cognitivo-conductual. En el momento del estudio el centro tenía capacidad para 35 internos.

\subsection{Participantes}

El presente estudio forma parte de uno más amplio en el que se evaluó la reincidencia de todos los internos que habían sido ingresados en el centro desde la fecha de su apertura en 1986 hasta julio de 1999. Todos los participantes en ese estudio eran menores que cumplían medidas de centro cerrado. 
Después de aplicar criterios de exclusión (sujetos de fuera de Cataluña o que habían fallecido) se obtuvo para el estudio más amplio una muestra de 224 sujetos varones. La edad en el momento de su desinternamiento estaba en el rango de 12 a 22 años. 68\% cumplían por delitos contra la propiedad y $32 \%$ por delitos contra las personas. El $46 \%$ ya había sido objeto de algún expediente judicial con anterioridad al ingreso en el centro, lo que muestra el alto nivel de conducta antisocial que mostraban los sujetos de la muestra.

Como el programa de tratamiento objeto de evaluación no empezó a aplicarse hasta unos años después de la apertura del centro, sólo fueron seleccionados para nuestra muestra los sujetos que ingresaron cuando el programa estaba ya aplicándose (a partir del 1 de Marzo de 1996).

Estos fueron 67 sujetos, pero 7 de ellos fueron excluidos por tener un nivel cultural excesivamente bajo para poder hacer este programa Así la muestra del presente estudio la constituyen 60 sujetos (26 que siguieron el programa en más del $75 \%$ de las sesiones y 34 que no lo siguieron) con características semejantes a la muestra del estudio más amplio. En concreto el grupo participante tenía una edad media en el momento del desinternamiento de 17,7 (DT=1,5) años de edad (rango entre 15 y 22 años). La edad promedio de dicho grupo de su primer expediente era de 13,9 (DT=2,1) con un rango entre 10 y 17 años.

El grupo no participante tenía unas características muy similares al anterior: 17,2 (DT=1,0) años de edad media en el momento de abandonar el centro (rango entre 15 y 20 años) y una edad media del primer expediente de 13,5 (DT=2,4) con un rango entre los 8 y los 17 años.

El seguir o no seguir el programa era aleatorio en función del momento del internamiento. Cuando había internos suficientes ( 7 u 8 personas) se iniciaba un grupo de tratamiento hasta que una vez finalizado se iniciaba otro. Así algunos internos no tuvieron tratamiento al haber ingresado al centro mientras se estaba desarrollando un programa con internos que habían ingresado anteriormente. Ninguno de los sujetos del grupo control había estado expulsado del programa o lo había abandonado de manera voluntaria. Los jóvenes del grupo de control no habían podido hacer el programa debido a las fechas de internamiento en el centro dado que, tal como hemos comentado, los 
grupos eran cerrados y no se podía incorporar ningún miembro hasta que se iniciaba un nuevo grupo.

\subsection{Programa de tratamiento}

El programa básico de competencia psicosocial constaba de 85 sesiones. Estaba constituido por las técnicas siguientes: Entrenamiento Estructurado en Habilidades Sociales (Goldstein et al., 1976), programa de pensamiento lateral (De Bono, 1985), la Técnica de Solución de Problemas (Spivack et al., 1976) y el Programa de Mejora de la autoestima (Hernández y García, 1993). Estas técnicas fueron adaptadas a la población local. Se hacían cinco sesiones a la semana de una hora cada una, de manera que la duración total era ligeramente superior a los cuatro meses. Cuando había suficientes sujetos se iniciaba de nuevo el programa. Los grupos se constituían con 7- 8 jóvenes y eran conducidos por 2 educadores entrenados en el uso de las técnicas descritas.

\subsection{Variables de estudio}

Nuestra variable independiente fue el haber seguido el tratamiento. Así, se establecieron dos grupos de sujetos: experimental, con los internos que habían realizado el programa, y control, con aquellos que no lo habían realizado.

Respecto a la variable dependiente de reincidencia, se usó como criterio el haber tenido una reincidencia grave en los 17 meses de seguimiento que se contaban desde el momento que el interno era liberado del centro. Así, para cada interno se determinaba una fecha concreta correspondiente a los 17 meses después del desinternamiento para determinar si en dicho periodo había reincidido. Se consideró reincidencia grave el haber vuelto a ser internado en un centro cerrado o en una prisión de adultos.

\subsection{Procedimiento}

Los datos de los participantes, referentes al seguimiento o no del programa de tratamiento y la fecha de desinternamiento, fueron recogidos de los archivos del mismo centro. Los datos de reincidencia fueron recogidos de la bases de datos de las 
direcciones generales de la Generalitat de Cataluña encargadas de la ejecución de penas tanto de menores como de adultos.

En el estudio comparamos los porcentajes de sujetos que habían reincidido en los respectivos grupos de los que participaron en el programa y de los que no participaron. Estas comparaciones se hicieron mediante la prueba de Chi cuadrado.

\section{Resultados}

La tabla 1 muestra los porcentajes en reincidencia para los sujetos que habían participado en el programa básico de competencia psicosocial y para los que no lo habían hecho. Como se puede observar, los porcentajes de internos que reincidieron son más elevados en el grupo de internos que no participó en el programa si bien las diferencias no fueron significativas.

Tabla 1. Número de sujetos y porcentajes de reincidencia en relación a la participación en el programa de competencia psicosocial.

\begin{tabular}{|c|c|c|c|}
\hline Participación en el programa & \multicolumn{3}{|c|}{ Reincidencia } \\
\hline & $\mathbf{N}$ & $\mathbf{N}$ & $\%$ \\
\hline SI & 26 & 5 & 19,0 \\
\hline NO & 34 & 12 & 35,0 \\
\hline Chi $^{2}$ & & & 1,87 NS \\
\hline
\end{tabular}

\section{Discusión}

\subsection{Conclusiones}

Estábamos particularmente interesados en evaluar los posibles efectos del programa básico de competencia psicosocial que fue aplicado en un centro de internamiento de jóvenes delincuentes. Como indicador seleccionamos la reincidencia como la media más idónea para evaluar los efectos a largo plazo del tratamiento (Grietens \& Hellinck, 2004). 
Para ello, escogimos un indicador de reincidencia grave, un nuevo internamiento en un centro de justicia juvenil o en una prisión, dentro de los 17 meses después de haberse producido la libertad del sujeto, ya que este era el tiempo mínimo de seguimiento que tenían los internos. Así podíamos conseguir un periodo de seguimiento común para todos los internos y un criterio de reincidencia objetivo.

Un estudio más amplio sobre la reincidencia con todos los internos que habían cumplido una medida de internamiento en el C.E. l'Alzina (Forcadell, Camps, Rivarola \& Pérez, 2004) mostró que el 77 \% de los desinternados habían tenido algún tipo de reincidencia y que el $31 \%$ cumplía el criterio de reincidencia grave ya que a los 17 meses del desinternamiento había vuelto a ser internado. Estas altas tasas de reincidencia no serían sorprendentes dado que los sujetos que cumplen en este centro reúnen todos o la mayoría de factores de riesgo asociados con la reincidencia. Diversos estudios muestran que la juventud de los delincuentes y la grave trayectoria criminal de estos son fuertes indicadores de reincidencia potencial (Benda, Corwyn \& Toombs, 2001; Duncan, Kennedy \& Patrick., 1995; Gendreau, Little \& Goggin, 1996; Minor, Hartmann \& Terry, 1997). Como mínimo estas dos características las cumplían casi todos los internos.

Cuando consideramos el principal objetivo de nuestro estudio, hemos observado que los sujetos que habían participado el programa de competencia psicosocial mostraban tasas de reincidencia más bajas que los que no habían participado. Es especialmente relevante el hecho que el $35 \%$ de los sujetos que no habían hecho el programa volvían a estar internados a los 17 meses después de su liberación. Por el contrario, el porcentaje de los que sí habían seguido el programa era manifiestamente más bajo (19\%). A pesar de la no significación estadística, posiblemente por el reducido número de sujetos, la diferencia en tasa de reincidencia, 16 puntos, es alta y superior a las que se han encontrado en la mayoría de estudios sobre la efectividad de los programas cognitivo conductuales (Landerberger \& Lipsey, 2005; Tong \& Farrington, 2006). No obstante, en el metaanálisis de Garrido et al (2006) sobre los programas de tratamiento para delincuentes graves en centros de seguridad aparecen 4 estudios con niveles de eficacia similares y 5 de ellos con niveles de eficacia superiores.

La clara reducción de la reincidencia encontrada podría ser explicada por el Modelo R-N-R (Andrews et al., 1990) que propone que las intervenciones son más 
eficaces cuando se aplican a delincuentes con niveles de riesgo de reincidencia más altos. Esta previsión quedaría avalada por Lipsey \& Howell (2012) ya que los datos de su metaanálisis indican que el grado de eficacia de los programas es más elevado cuando se aplican a delincuentes juveniles de alto riesgo.

\subsection{Limitaciones}

Creemos que nuestro estudio presenta algunas fortalezas y también ciertas limitaciones. Entre estas últimas podríamos citar el hecho de que los grupos no fueran configurados al azar y de que los resultados fueran obtenidos hace algunos años. Creemos que la primera limitación podría haber sido mitigada por la forma real de configuración de los grupos. Dado que el número de grupos de tratamiento que podían funcionar al mismo tiempo era limitado por la disponibilidad de profesionales y espacios, se constituía un grupo cuando habían ingresado suficientes internos para poderse formar y no se constituía otro hasta la finalización del tratamiento con el primero y hubiera de nuevo suficientes internos para formar otro grupo. Así, tal como se ha explicado en el apartado de participantes, la inclusión o no de un sujeto en un grupo de tratamiento dependía de si en el momento en el que ingresara en el centro había que formar un grupo de tratamiento, lo cual sí que dependía del azar. De hecho los dos grupos fueron muy semejantes en cuanto a edad en el momento de desinternamiento y cuando tuvieron el primer expediente delictivo.

\subsection{Implicaciones}

La posible limitación referente a que los resultados del estudio fueran obtenidos hace algunos años pensamos que no anularía la validez de los mismos ya que son pocos los estudios sobre el tema realizados en nuestro país. Precisamente esto sería uno de los puntos fuertes del trabajo ya que aporta datos concretos sobre la posible eficacia de un programa de tratamiento.

Como se ha dicho en la introducción, la inmensa mayoría de los estudios sobre el tema han sido realizados en países anglosajones. Así, pensamos que otro de los puntos fuertes, es que el estudio se ha realizado en un país mediterráneo cuyos 
resultados coinciden con los provenientes de las investigaciones de los países anglosajones. Así estos resultados colaboraría a dar soporte a que "alguna cosa funciona" en lugar del "nada funciona" y colaboran a confirmar el efecto positivo de los programas cognitivo conductuales.

Consideramos que los resultados del presente estudio son útiles al centro donde el programa se ha realizado porque ofrece los primeros datos sobre la efectividad del programa sobre la vida de los internos fuera de la institución. Los datos apuntan claramente a que la participación en el programa de competencia psicosocial estaría asociada con una baja tasa de reincidencia. Este hecho podría ser muy motivador para los profesionales del centro.

Por último, los resultados encontrados pueden actuar como estimulo para que se mantenga y se incremente el uso de este tipo de programas en las intervenciones rehabilitadores con delincuentes juveniles en tanto en Cataluña como en el resto de España.

\section{Agradecimientos}

A Alfredo Gil, que fue pionero en la implementación de programas cognitivo conductuales en la Justicia Juvenil de Catalunya con la confección e impulso de este y otros programas. Sin su trabajo este artículo no hubiera sido posible. A todos los profesionales del Centro Educativo l'Alzina que con su entusiasmo y dedicación hicieron posible la aplicación de este y otros programas.

\section{Financiación}

La realización del presente estudio contó con una ayuda del Centro de Estudios y Formación Especializada (CEJFE) del Departamento de Justicia de la Generalitat de Cataluña en 2003.

\section{Referencias}

Andrews, D.A., Bonta, J., \& Hoge, R.D. (1990). Classification for effective rehabilitation: Rediscovering psychology. Criminal Justice and Behavior, 17, $19-52$.

Andrews, D.A., Zinger, I, Hoge, R.D., Bonta, J., Gendreau, T. \& Cullen, F.T. (1990). Does correctional treatment work? A clinically relevant and psychologically informed meta-analysis, Criminology, 28, 419-429. 
Antonowicz, D.H., \& Ross, R.R. (1994). Essential components of successful rehabilitation programs for offenders. International Journal of Offender Therapy and Comparative Criminology, 38, 115-134.

Benda, B.B., Corwyn, R.F. \& Toombs, N. J. (2001). Recidivism among adolescents serious offenders: Prediction of entry into correctional system for adults. Criminal Justice and Behavior, 28, 588-613.

Cann, J., Falshaw, L. \& Friendship, C (2005). Understanding "What Works": accredited cognitive skills programmes for young offenders. Youth Justice, 5, 165-179.

De Bono, E. (1985). The CORT thinking program. En J. Segal, S. Chidman \& R. Glaser (Eds), Thinking \& learning skills. Hillsdale, NJ: Eribaum.

Duncan, R.D., Kennedy, W.A., y Patrick, C.J. (1995) Four-Factor model of recidivism in male juvenile offenders. Journal of Clinical Child psychology, 24, 250-257.

Forcadell, A., Camps, C., Rivarola, P. \& Pérez, J. (2004). Evaluación de la reincidencia de los menores desinternados del centro educativo L'Alzina. Invesbreu, $\mathrm{n}^{\mathbf{o}} 26$, 6-8.

Galbraith, R.E. \& Jones, T.M. (1976). Moral reasoning. Minneapolis, MN: Greenhaven Press.

Garrido, V. (1989). El modelo cognitivo en la prevención y tratamiento de la delincuencia,. Aplicación en delincuentes españoles. Delincuencia, 1, 236-248.

Garrido, V., Morales, L.A. \& Sánchez-Meca, J. (2006). What works for serious juvenile offenders? A systematic review. Psicothema, 18, 611-619.

Garrido, V., Redondo, S. \& Pérez, E. (1989). El tratamiento de delincuentes institucionalizados: el programa de competencia psicosocial en la prisión de jóvenes de La Trinidad de Barcelona. Delincuencia, 1, 38-57.

Gendreau, P, Little, T. \& Goggin, C (1996). A Meta-analysis of the predictors of adult offender recidivism: What works! Criminology, 34, 575- 607.

Goldstein, A.P., Sprafkin, R. \& Gershaw , N., (1976). Skill training for community living. New York: Pergamon.

Grietens, H., \& Hellinckx, W. (2004). Evaluating effects of residential treatment for juvenile offenders by statistical metaanalysis: A review. Aggression and Violent Behavior, 9, 401-415.

Hernández, P., \& García, M.D. (1993). PIELE: Programa instruccional para la educación y la liberación emotiva: Madrid: TEA. 
Hollin, C.R., McGuire, J., Hounsome, J., Hatcher, R.M., Bilby, C.A.L. \& Palmer, E.J. (2008). Cognitive skills behavior programs for offenders in the community: A reconviction analysis. Criminal Justice and Behavior, 35, 269-283.

Kilias, M., Redondo, S. \& Sarnecki, J. (2012). European Perspectives. En R. Loeber \& D. Farrington (Eds.). From juvenile delinquency to adult crime: Criminal careers, justice policy and prevention. New York: Oxford University Press.

Landenberger, N.A \& Lipsey, M.W. (2005). The positive effects of cognitivebehavioral programs for offenders: A meta-analysis of factors associated with effective treatment. Journal of Experimental Criminology, 1, 451-476.

Lipsey, M.W., \& Howell, J.C. (2012). A broader view of evidence based programmes reveals more options for state juvenile justice systems. Criminal and Public Policy, 11, 515-523.

Martinson, R. (1974). What works? - questions and answers about prison reform. Public Interest, 1, 22-54.

Meichenbaum, D. (1987). Manual de inoculación de estrés. Barcelona: Martínez Roca.

Minor, K.I., Hartmann, D.J., y Terry, S. (1997). Predictors of juvenile court actions and recidivism. Crime \& Delinquency, 43, 3, 328-344.

Redondo, S. (2008). Manual para el tratamiento psicológico de los delincuentes. Madrid: Pirámide.

Redondo, S., Álvarez, M., Antequera, M., Cano, A. \& Martínez, A. (2010). Evaluación de una intervención psicológica con delincuentes juveniles, Boletin Criminológico, 124, 1-4.

Redondo, S., Garrido, V., \& Sánchez-Meca, J. (1997). What works in correctional rehabilitation in Europe: A meta-analytical review. En S. Redondo, V. Garrido, J. Pérez \& R. Barberet (Eds.) Advances in psychology and law. Berlín : Walter de Gruyter

Ross, R.R., Fabiano, E.A., \& Garrido, V, (1990). Pensamiento prosocial: El modelo cognitivo para la prevención y el tratamiento de la delincuencia Delincuencia, 1 (Monográfico), 1-116.

Ross, R., \& Fabiano, E.A. (1985). Time to think: A cognitive model of delinquency prevention and offender rehabilitation. Johnson City, TN: Institute of Social Sciences and Arts.

Spivack, G., Platt, J.J \& Shure, M.B., (1976). The solving-problem approach to adjustment: A guide to research and intervention. San Francisco: Jossey-Bass. 
Tong, L.S., \& Farrington, D (2006). How effective is the "Reasoning and Rehabilitation" programme in reducing reoffending? A meta-analysis of evaluations in four countries. Psychology, Crime and Law, 12, 3-24.

Van Voorhis, P., Spruance, L., Johnson Listwan, S., Ritchie, N. \& Seabrook, R. (2004). Results of the Georgia Cognitive Skills experiment: A replication of reasoning and rehabilitation. Criminal Justice and Behavior. 31, 282-305.

Antoni Forcadell es doctor en Psicología por la Universidad Autónoma de Barcelona y actualmente es psicólogo del Centre Penitenciario Abierto de Girona y profesor asociado en la Facultad de Derecho de la Universidad Pompeu Fabra. Es profesor de Prevención y Tratamiento de la Delincuencia en el grado de Criminología y Políticas Públicas de Prevención. Su tema de investigación está relacionado con el tratamiento de la delincuencia.

Jorge Pérez es doctor en Psicología por la Universidad Autónoma de Barcelona y es profesor titular en la Facultad de Ciencias de la Salud y de la Vida de la Universidad Pompeu Fabra. Actualmente es el director de la Oficina Educativa de dicho centro del que fue decano entre 1999 y 2011. Es profesor de Psicología en los grados de Biología Humana, de Medicina y de Criminología en la UPF. Su tema de investigación estuvo centrado en las variables de predisposición a la delincuencia. Actualmente está centrado en la investigación educativa. 